\section{Ecological chaos}

SIR - In his important contribution, Stone $^{1}$ analyses one-dimensional maps that exhibit period-doubling reversals using an additive constant immigration term $(\lambda)$. Taking the Ricker's equation as a reference model, he shows that for increasing $\lambda$, reversal takes place and chaos becomes more difficult to observe. As a consequence, he concludes that chaotic dynamics in ecology may result from using simple models than from the real world.

We wish to raise some questions. One is how the constant 'immigration' term can be large enough to dominate densitydependence. Another involves the explicit introduction of space in ecological models $^{2-6}$. Spatial degrees of freedom are able to generate complex dynamics together with ordered patterns ${ }^{2-4}$. If dispersal between patches is introduced, chaos is common for almost all parameter combinations, both for single species ${ }^{4}$ and for two-species models involving LotkaVolterra $^{3}$ or host-parasitoid ${ }^{2}$ models. We have shown ${ }^{4}$ that the critical boundaries separating stable states from oscillations and chaos in the parameter space are strongly shifted even when very small lattices and diffusions are used.
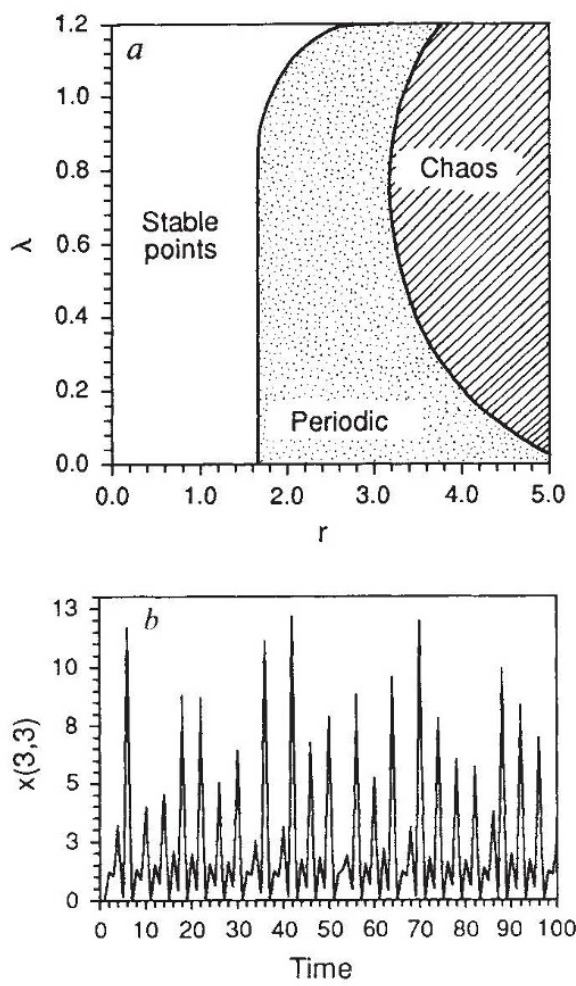

a, State space for the spatial counterpart of Stone's model. We use a coupled map lattice $^{3,4}$. Lattice size, $6 \times 6$; diffusion coefficient coupling nearest neighbours, $D=0.05$. There is a well-defined region characterized by chaotic dynamics. Compare with Fig. 2 of Stone's paper ${ }^{1} . b$, Local temporal series for the coupled map version of Stone's model for $\lambda=1$ and $r=5$.
We have now studied a $6 \times 6$ lattice model with diffusion coefficient $D$ between the nearest patches. The results obtained for $D=0.05$ are summarized in the figure $(a)$. As can be seen, even in such a small spatial domain, chaos emerges for enough high growth rates $(r)$, even for very high $\lambda$ values. With $D=0.1$, most of the previous periodic points become chaotic. The figure $(b)$ shows an example of local chaotic oscillations obtained for $\lambda=1$ and $r=5$, whereas Stone reported only periodic orbits. As a consequence of diffusion, space starts to dominate the dynamics.

Concerning the stability properties, global populations in presence of spatial chaos have been shown to be nearly constant (stable $)^{3-6}$. The origin of this stability is that spatial chaos leads to short correlation lengths and so patches tend to be independent. As a consequence, the global extinction probability is very small $^{3,5}$. Stone's results will encourage those working on chaos to carry the discussion further, and to examine the robustness of chaotic phenomena in ecology.

Ricard V. Solé

Department de Física i Enginyeria Nuclear, Universitat Politècnica de Catalunya

08034 Barcelona

Jordi Bascompte

Department d'Ecologia,

Universitat de Barcelona,

08028 Barcelona, Spain

1. Stone, L. Nature $365,617-620$ (1993).

2. Hassell, M. P. et al. Nature 353, 255-258 (1991)

3. Solé, R. V. \& Valls, J. J. theor. Biol. 155, 87-102 (1992).

4. Bascompte, J.\& Solé, R. V. J. Anim. Ecol. (in the press)

5. Allen, J. C. etal. Nature 364, 229-232 (1993)

6. Collins, H. N. etal.J.Anim. Ecol. 6, 735-748(1992).

STONE REPLIES - The reversal of period doubling cascades is a fundamental, if not inevitable, phenomenon common to many non-linear dynamical systems and strikingly apparent in even the simplest of difference equations ${ }^{1-3}$. Although I commented and possibly over-emphasized the observation that period-doubling reversals may suppress chaos in simple models ${ }^{3}$, Solé and Bascompte imply that the phenomenon is absent in more complex systems. After criticizing an allegedly large immigration parameter in one of my examples - the Ricker map (the parameter was in fact a negligible perturbation) - Solé and Bascompte escalate the effects of this ecological process by allowing populations to migrate between a set of patches as well. But, unlike the Ricker map and more complex non-linear equations ${ }^{4}$, in their spatially extended model chaos occurs over a significant region of parameter space (but only at very high and possibly unrealistic intrinsic growth rate levels).

Are these findings in conflict with my general thesis? A preliminary examination of the spatial model of Solé and
Bascompte uncovers the existence of a complicated structure of period doubling and halving bifurcations, particularly in the parameter regime they classify as "periodic". This seems to reinforce rather than to contradict my basic argument. As it happens, there is a simple explanation as to why the Ricker model tends to suppress chaos more than the patch model, and it appears to have little connection with factors that concern spatial effects. My analysis of the Ricker model ${ }^{3}$ rested on a key working assumption advanced by McCallum $^{5}$ : given that in the real world there are numerous subpopulations that rely on refuges for protection or an influx of individuals by immigration, these organisms must have a 'floor' $(\lambda)$ below which the population level never falls. It is this floor which enhances the initiation of a period-doubling since, in the terminology of my article, it forms a 'plateau' in the equation's associated return map ${ }^{3}$. However, for Solé and Bascompte's patch model, spatial diffusion enables populations to fall far below the set floor $(\lambda)$ (see their figure $b$ in which the population falls to zero when $\lambda=1$ ), and without preventative measures may even create absurd 'negative' populations. These effects destroy the 'plateau' in the model's return map, and thereby inhibit perioddoubling reversals.

To test this possibility further, I incorporated McCallum's suggestion of a population floor in Solé and Bascompte's spatial model. The results are similar in character to that of the Ricker map chaos being markedly suppressed together with the appearance of perioddoubling reversals. Thus, the findings of Solé and Bascompte appear to differ not so much because of the novel spatial dimension incorporated into their model, but more likely because they violate a key working assumption.

My focus on the Ricker map was deliberate, for it provides a dramatic demonstration of the reversal of a period doubling cascade. This intrinsic phenomenon escaped the attention of a generation of mathematical biologists, who have been studying the dynamics of the Ricker map "under the microscope" for well over 20 years. In a similar way, periodic-doubling reversals are understood to be widespread in more general nonlinear systems. Should readers have any lingering doubts concerning this possibility, they will find further convincing evidence in the recent and intriguing studies of Dawson et al. ${ }^{1,2}$.

\section{Lewi Stone}

Department of Zoology

Tel Aviv University

Tel Aviv 69978, Israel

1. Dawson, S. P. et al. Phys. Lett. A162, 249-254 (1992)

2. Dawson, S. P. et al. Phys. Rev. E48, 1676-1682 (1993)

3. Stone, L. Nature 365, 617-620 (1993).

4. Sprott, J. C. Phys. Lett. A173, 21-24 (1993)

5. McCallum, H. I. J. theor. Biol. 154, 277-284 (1992). 